International Mathematical Forum, 1, 2006, no. 9, $403-420$

\title{
SOLVING FLEXIBLE FLOW-SHOP PROBLEMS BY LPT AND LN SCHEDULING ALGORITHMS
}

\author{
Tzung-Pei Hong \\ Department of Electrical Engineering \\ National University of Kaohsiung \\ Kaohsiung, 811, Taiwan, R.O.C. \\ tphong@nuk.edu.tw
}

\section{Gwoboa Horng, Pei-Ying Huang and Chien-Lung Wang}

\author{
Department of Computer Science \\ National Chung-Hsing University \\ Taichung, 40227, Taiwan, R.O.C. \\ gbhorng@cs.nchu.edu.tw \\ s9156053@cs.nchu.edu.tw \\ phd9004@cs.nchu.edu.tw
}

\begin{abstract}
Scheduling is an important process widely used in manufacturing, production, management, computer science, and so on. Appropriate scheduling not only reduces manufacturing costs but also reduces possibilities for violating due dates. Finding good schedules for given sets of jobs can thus help factory supervisors effectively control job flows and provide solutions for job sequencing. In simple flow shop problems, each machine operation center includes just one machine. If at least one machine center includes more than one machine, the scheduling problem becomes a flexible flow-shop problem. Flexible flow shops can thus be thought of as generalizations of simple flow shops. In the past, Sriskandarajah and Sethi proposed a heuristic algorithm for solving flexible flow-shop problems of two machine centers. In this paper, we extend their algorithm to solve flexible flow-shop problems of more than two machine centers. The heuristic LN algorithm is adopted as the kernel for achieving this purpose. Since this problem is NP-complete, optimal solutions seem unnecessary especially when the number of jobs is large.
\end{abstract}

AMS Subject Classification Code: 90B35

Keywords: scheduling, flexible flow shop, LPT scheduling, LN scheduling 


\section{Introduction}

Tzung-Pei Hong et al

In simple flow-shop problems, each machine center has just one machine. If at least one machine center has more than one machine, the problem is called a flexible flow-shop problem [2]. Flexible flow shops are thus generalizations of simple flow shops. Scheduling jobs in flexible flow shops is considered an NP-complete problem [1][5].

In the past, Sriskandarajah and Sethi proposed a heuristic algorithm for solving flexible flow-shop problems of two machine centers. In this paper, we extend their algorithm to solve flexible flow-shop problems of more than two machine centers. A scheduling algorithm, which combines the LPT and LN algorithms, is thus proposed for the flexible flow shops with more than two machine centers. The LPT method is first used to assign jobs to each machine group (flow shop). The LN algorithm is then used to deal with job sequencing. Experimental results show that our proposed method can save much computational time than the optimal approach although the obtained makespans may be a little larger than the optimal solutions. It is thus more suitable for real applications.

The remainder of this paper is organized as follows. Some related scheduling algorithms are reviewed in Section 2. The assumptions and notation used in this paper are described in Section 3. An algorithm for scheduling on a flexible flow shop with more than two machine centers is proposed in Section 4. An example to illustrate the proposed scheduling algorithm is given in Section 5. Experiments for verifying the performance of the proposed algorithm are described in Section 6 . Finally, conclusions are given in Section 7.

\section{Review of Some Related Scheduling Algorithms}

As mentioned above, flexible flow-shop problems are NP-complete. No algorithms except exhaustive search have ever been provided for finding optimal solutions. In the past, Sriskandarajah and Sethi proposed a heuristic algorithm to solve the problem of two machine centers, and the completion time of the derived schedules was close to the optimum. In this paper, we generalize it and adopt the LN algorithm [4] to solve the flexible flow-shop problems of more than two machine centers. Some related scheduling algorithms are first introduced as follows.

\subsection{The LPT Scheduling Algorithm}

The discovery of scheduling algorithms for a set of independent tasks with arbitrary execution time and an arbitrary number of processors is a classic sequencing problem of wide interest and application. Among the scheduling algorithms 
proposed, the LPT (Longest-Processing-Time-first) scheduling algorithm is the simplest one and is widely used in many real-world situations.

Given a set of $n$ independent tasks ( $T_{1}$ to $T_{n}$ ), each with arbitrary execution time $\left(t_{1}\right.$ to $\left.t_{n}\right)$, and a set of $m$ homogeneous processors or machines ( $P_{1}$ to $P_{m}$ ), the LPT scheduling algorithm assigns the task with the longest execution time (among those not yet assigned) to a free processor whenever this processor becomes free. For cases when there is a tie, an arbitrary tie-breaking rule can be assumed. The algorithm is described as follows.

The LPT scheduling algorithm:

Input: A set of $n$ tasks, each with arbitrary processing time, and a set of $m$ processors.

Output: A schedule and the final finishing time of all the tasks.

Step 1: Sort the tasks in a descending order according to the processing time.

Step 2: Initialize the current finishing time of each processor to zero.

Step 3: Assign the first task in the task list to the processor with the minimum finishing time.

Step 4: Set the new finishing time of the processor = the old finishing time of the processor + the execution time of the task.

Step 5: Remove the task from the task list.

Step 6: Repeat Steps 3 to 5 until the task list is empty.

Step 7: Among the finishing time of the processors, choosing the longest as the final finishing time.

The finishing time by the LPT scheduling algorithm is in general not minimal. The computational time spent by the LPT scheduling algorithm is, however, much lower than that by an optimal scheduling algorithm.

\subsection{The LN Scheduling Algorithm}

The LN algorithm [4] was proposed to schedule job sequencing for a flow shop with more than two machines. Given a set of $n$ independent jobs, each having $m$ ( $m>2)$ tasks $\left(T_{11}, T_{21}, \ldots, T_{m 1}, T_{12}, T_{22}, \ldots, T_{(m-1) n}, T_{m n}\right)$ that must be executed in the same sequence on $m$ machines $\left(P_{1}, P_{2}, \ldots, P_{m}\right)$, the LN scheduling algorithm seeks a nearly minimum completion time of the last job. This algorithm is stated as follows.

The LN scheduling algorithm:

Input: A set of $n$ jobs, each having $m(m>2)$ tasks executed respectively on each of $m$ machines,

Output: A schedule with a nearly minimum completion time of the last job. 
Step 1: For each job $i$, compute the average execution time of each task, $T_{i}^{a v g}=\left(\sum_{j=1}^{m} t_{j i}\right) / m$, where $t_{j i}$ is the execution time needed for the $j$-th task of the $i$-th job.

Step 2: Sort the jobs in a descending order of $T_{i}^{a v g}$.

Step 3: Select the first two jobs from the sorted list in Step 2 and generate the two possible partial schedules for the two jobs.

Step 4: Calculate the total completion time of each partial schedule generated in Step 3.

Step 5: Keep the partial schedule with a smaller total completion time.

Step 6: Set $k=3$.

Step 7: Repeat Steps 8 to 11 until $k>n$.

Step 8: Select the $k$-th job from the sorted list in Step 2 and generate the $k$ possible partial schedules by inserting the $k$-th job in the partial schedule kept in the last run.

Step 9: Calculate the total completion time of each partial schedule generated in Step 8.

Step 10: Keep the partial schedule with the minimum total completion time.

Step 11: Set $k=k+1$

After Step 11, scheduling is finished and a completion time has been found.

\subsection{Sriskandarajah and Sethi’s Scheduling Algorithm}

Sriskandarajah and Sethi proposed a heuristic algorithm [7] for solving the flexible flow-shop problem of two machine centers. Sriskandarajah and Sethi decomposed the problem into the following three subproblems and solved each heuristically:

Part 1: Form the machine groups, each of which contains a machine from each center.

Part 2: Use the LPT method to assign jobs to each machine group (flow shop).

Part 3: Deal with job sequencing and timing using the Johnson algorithm.

In this paper, we will extend their approach to solve the flexible flow-shop problems of more than two machine centers.

\section{Assumptions and Notation}

Assumptions and notation used in this paper are described in this section.

Assumptions: 
- Jobs are not preemptive.

- Each job has $m(m>2)$ tasks with processing times, executed respectively on each of $m$ machine centers.

- Each machine center has the same number of homogeneous machines.

Notation:

$n$ : The number of jobs.

$m$ : The number of tasks in each job.

$m c_{i}$ : The $i$-th machine center, $i=1,2, \ldots, m$.

$p$ : The number of machines in each machine center.

$F_{i}$ : The $i$-th allocated machine group (flow shop), $i=1$ to $p$.

$F_{j i}$ : The $j$-th machine of the $i$-th flowshop $F_{i}, j=1$ to $m$.

$f_{i}$ : The completion time of the $i$-th flowshop.

$f_{j i}$ : The completion time of the $j$-th machine in the $i$-th flowshop.

$T_{j i}$ : The $j$-th task for the $i$-th job, $j=1$ to $m$ and $i=1$ to $n$.

$t_{j i}$ : The execution time of $T_{j i}$.

$t t_{i}$ : The total execution time of the $i$-th job.

$Q_{F_{i}}$ : The group of jobs executed on the $i$-th flowshop.

$f f:$ The final completion time of the whole schedule.

\section{The Flexible Flow-shop Scheduling Algorithm by LPT and LN}

Here, we generalize Sriskandarajah and Sethi's approach to solve flexible flow-shop problems of more than two machine centers. The flexible flow-shop algorithm for more than two machine centers is based on the LPT [3] and the LN [4] algorithms to manage job scheduling. The flexible flow-shop algorithm is decomposed into three parts as Sriskandarajah and Sethi's approach was. The first part forms the machine groups, each of which contains a machine from each center [6][9]. The second part uses the LPT method to assign jobs to each machine group (flow shop). The third part deals with job sequencing and timing using the LN algorithm. The proposed flexible flow-shop algorithm is stated below.

A LPT_LN flexible flow-shop algorithm:

Input: A set of $n$ jobs, each having $m(m>2)$ tasks, to be executed respectively on each of $m$ machine centers with $p$ homogenous machines.

Output: A schedule with a completion time.

Part 1: Forming the machine groups. 
Step 1: Form $p$ machine pairs, each of which contains one machine from each machine center. Each machine pair can be thought of as a simple flow shop $F_{1}, F_{2}, \ldots, F_{p}$.

Step 2: Initialize the completion time of each flow shop $f_{1}, f_{2}, \ldots, f_{p}$ to zero.

Part 2: Assigning jobs to machine groups.

Step 3: For each job $J_{i}, 1 \leq i \leq n$, find its total execution time $t t_{i}=t_{1 i}+t_{2 i}+\ldots+t_{m i}$.

Step 4: Sort the jobs in descending order of processing time $t t_{i}$; if any two jobs have the same $t t_{i}$ values, sort them in an arbitrary order.

Step 5: Find the flow shop $F_{i}$ with the minimum processing time $f_{i}$ among all the flow shops; if two flowshops have the same minimum $f_{i}$ value, choose one arbitrarily.

Step 6: Assign the first job $J_{i}$ in the sorted list to the chosen flow shop $F_{i}$ that has the minimum completion time $f_{i}$ among all $p$ flow shops.

Step 7: Add the total time $t t_{i}$ of job $J_{i}$ to the needed total time of the chosen flow shop, $F_{i}$; that is:

$$
f_{i}=f_{i}+t t_{i}
$$

Step 8: Remove job $J_{i}$ from the job list.

Step 9: Repeat Steps 5 to 8 until the job list is empty.

After Step 9, jobs are clustered into $p$ groups and are allocated to the $p$ machine pairs (flow shops).

Part 3: Dealing with job sequencing in each flow shop

Step 10: For each job $i$, compute the average execution time of each task, $T_{i}^{a v g}=\left(\sum_{j=1}^{m} t_{j i}\right) / m$, where $t_{j i}$ is the execution time needed for the $j$-th task of the $i$-th job.

Step 11: Sort the jobs in a descending order of $T_{i}^{\text {avg }}$.

Step 12: Select the first two jobs from the sorted list in Step 11 and generate the two possible partial schedules.

Step 13: Calculate the total completion time of each partial schedule in Step 12.

Step 14: Keep the partial schedule with a smaller total completion time.

Step 15: Set $k=3$.

Step 16: Repeat Steps 17 to 20 until all the jobs in the sorted list are processed.

Step 17: Select the $k$-th job from the sorted list in Step 11 and generate the $k$ possible partial schedules by inserting the $k$-th job in the partial schedule kept in the last run.

Step 18: Calculate the total completion time of each possible partial schedule generated in Step 17.

Step 19: Keep the partial schedule with the minimum total completion time.

Step 20: Set $k=k+1$ and do Step 16 . 
Solving flexible flow-shop problems

Step 21: Denote the chosen schedule in $F_{i}$ as $Q_{F i}$ and set the final completion time of each flowshop $f_{i}=$ the completion time of the $m$-th machine $f_{m i}$ in $Q_{F i}$.

Step 22: Find the maximum final completion time $f f=\max _{i=1}^{p}\left(f_{i}\right)$ among the completion time of all the flowshops.

After Step 22, scheduling is finished and a total completion time ff has been found.

\section{An Example}

Assume eight jobs, $J_{1}$ to $J_{8}$, each having three tasks $\left(J_{1 i}, J_{2 i}, J_{3 i}\right)$, are to be scheduled via three operations. Each operation is executed by a machine at the corresponding machine center. Each machine center includes three homogeneous machines. Assume the execution times of these jobs are listed in Table 1. The algorithm proceeds as follows.

Table 1. Processing times for the eight jobs

\begin{tabular}{|c|c|c|c||}
\hline$J_{\text {ob }}$ & $t_{1 i}$ & $t_{2 i}$ & $t_{3 i}$ \\
\hline$J_{1}$ & 4 & 7 & 3 \\
\hline$J_{2}$ & 1 & 5 & 2 \\
\hline$J_{3}$ & 5 & 2 & 4 \\
\hline$J_{4}$ & 2 & 5 & 3 \\
\hline$J_{5}$ & 5 & 5 & 6 \\
\hline$J_{6}$ & 7 & 3 & 4 \\
\hline$J_{7}$ & 4 & 4 & 5 \\
\hline$J_{8}$ & 3 & 6 & 3 \\
\hline
\end{tabular}

Part 1: Forming the machine groups.

Step1: Form three machine groups, $F_{1}, F_{2}, F_{3}$, each of which is thought of as a three-machine flowshop. Without lose of generality, we may assume the flowshops are constructed as follows:

$$
\begin{aligned}
& F_{1} \rightarrow\left\{m_{11}+m_{12}+m_{13}\right\}, \\
& F_{2} \rightarrow\left\{m_{21}+m_{22}+m_{23}\right\}, \text { and } \\
& F_{3} \rightarrow\left\{m_{31}+m_{32}+m_{33}\right\},
\end{aligned}
$$

where $m_{i j}$ is the $i$-th machine in the $j$-th center.

Step 2: Initialize $f_{1}=f_{2}=f_{3}=0$, where $f_{i}$ is the initial completion time of $F_{i}$.

Part 2: Assigning jobs to machine groups. 
Step 3: For each job $J_{i}, i=1$ to 8 , find its total execution time $t t_{i}=t_{1 i}+t_{2 i}+t_{3 i}$. For example, the total processing time of job 1 is calculated as:

$$
t t_{1}=t_{11}+t_{21}+t_{31}=4+7+3=14 .
$$

The total processing times of the other jobs can be similarly found and the results are listed in Table 2.

Table 2. The total processing times of the eight jobs

\begin{tabular}{||c|c||}
\hline \hline$J_{o b}$ & total processing time $t_{i}$ \\
\hline$J_{1}$ & 14 \\
\hline$J_{2}$ & 8 \\
\hline$J_{3}$ & 11 \\
\hline$J_{4}$ & 10 \\
\hline$J_{5}$ & 16 \\
\hline$J_{6}$ & 14 \\
\hline$J_{7}$ & 13 \\
\hline$J_{8}$ & 12 \\
\hline
\end{tabular}

Step 4: Sort the jobs $J_{1}$ to $J_{8}$ in a descending order of the total processing time $\left(t t_{i}\right)$.

The following results can be obtained:

$$
\text { Job list }=\left\{J_{5}, J_{1}, J_{6}, J_{7}, J_{8}, J_{3}, J_{4}, J_{2}\right\} .
$$

Step 5: Find the minimum $f_{i}$ among all the flowshops $F_{1}$ to $F_{3}$. Since all the total processing time of flowshops is currently equal to zero, any arbitrary one can be chosen. Without lose of generality, assume $F_{1}$ is chosen.

Step 6: Assign the first job $J_{5}$ in the sorted list to the chosen flowshop $F_{1}$.

Step 7: Add the total processing time $t t_{5}$ of job $J_{5}$ to the needed total time of the chosen flowshop $F_{1}$. Thus:

$$
f_{1}=f_{1}+t t_{5}=0+16=16 .
$$

The results after allocating $J_{5}$ to the flowshop $F_{1}$ are shown in Table 3 .

Table 3. The flowshops with allocated jobs and total processing time

\begin{tabular}{||c|c|c||}
\hline Flowshop $_{i}$ & allocated jobs & total processing time \\
\hline$F_{1}$ & $J_{5}$ & 16 \\
\hline$F_{2}$ & None & 0 \\
\hline$F_{3}$ & None & 0 \\
\hline
\end{tabular}

Step 8: Remove the job $J_{5}$ from the job list. After $J_{5}$ is removed, the job list is then as follows:

$$
\text { Job list }=\left\{J_{1}, J_{6}, J_{7}, J_{8}, J_{3}, J_{4}, J_{2}\right\} .
$$

Step 9: Repeat Steps 5 to 8 until the job list is empty. After Step 9, jobs are clustered into three groups and are respectively allocated to the three 
flowshops. Results are shown in Table 4.

Table 4. The jobs in each flowshop

\begin{tabular}{||c|c||}
\hline \hline Flowshop $_{i}$ & Jobs allocated \\
\hline$F_{1}$ & $J_{5}, J_{3}, J_{2}$ \\
\hline$F_{2}$ & $J_{1}, J_{8}, J_{4}$ \\
\hline$F_{3}$ & $J_{6}, J_{7}$ \\
\hline
\end{tabular}

Part 3: Dealing with job sequencing in each flow shop.

Step 10: For each job $J_{i}, i=1$ to 8 , find the average execution time of each task. For example, the average total execution time of each task in job 1 is calculated as:

$$
T_{1}^{a v g}=\left(\sum_{j=1}^{3} t_{j 1}\right) / 3=2.67 .
$$

The average total execution time of the tasks in the other jobs can be similarly calculated and the results are listed in Table 5.

Table 5. The average execution time of a task in the eight jobs

Flow shop 1:

\begin{tabular}{||c|c|}
\hline \hline$J_{0} b_{i}$ & average execution time $T_{i}^{\text {avg }}$ \\
\hline$J_{2}$ & 2.67 \\
\hline$J_{3}$ & 3.67 \\
\hline$J_{5}$ & 5.33 \\
\hline
\end{tabular}

Flow shop 2:

\begin{tabular}{|c|c|}
\hline \hline$J_{o b}$ & average execution time $T_{i}^{\text {avg }}$ \\
\hline$J_{1}$ & 4.67 \\
\hline$J_{4}$ & 3.33 \\
\hline$J_{8}$ & 4 \\
\hline
\end{tabular}

Flow shop 3:

\begin{tabular}{||c|c|}
\hline \hline$J_{o b_{i}}$ & average execution time $T_{i}^{\text {avg }}$ \\
\hline$J_{6}$ & 4.67 \\
\hline$J_{7}$ & 4.33 \\
\hline
\end{tabular}

Step 11: Sort the jobs $J_{1}$ to $J_{8}$ in a descending order of the average execution time of tasks. The following results can be obtained:

Flow shop 1: Job list $=\left\{J_{5}, J_{3}, J_{2}\right\}$,

Flow shop 2: Job list $=\left\{J_{1}, J_{8}, J_{4}\right\}$, and 
Flow shop 3: Job list $=\left\{J_{6}, J_{7}\right\}$.

Step 12: For each flow shop, select the first two jobs from the sorted list in Step 11 and generate the two possible partial schedules. Results are shown as follows:

Flow shop1: possible partial schedules $=\left\{\begin{array}{l}J_{5}, J_{3} \\ J_{3}, J_{5}\end{array}\right\}$,

Flow shop2: possible partial schedules $=\left\{\begin{array}{l}J_{1}, J_{8} \\ J_{8}, J_{1}\end{array}\right\}$, and

Flow shop3: possible partial schedules $=\left\{\begin{array}{l}J_{6}, J_{7} \\ J_{7}, J_{6}\end{array}\right\}$.

Step 13: Calculate the total completion time of each partial schedule in Step 12. Results are shown in Table 6.

Step 14: Keep the partial schedule with a smaller total completion time. Results are shown as follow:

Flow shop1: chosen partial schedule $=\left\{J_{5}, J_{3}\right\}$,

Flow shop2: chosen partial schedule $=\left\{J_{8}, J_{1}\right\}$, and

Flow shop3: chosen partial schedule $=\left\{J_{7}, J_{6}\right\}$.

Step 15: Set $k=3$.

Step 16: Repeat Steps 17 to 20 until all the jobs in the sorted list in each flow shop are processed. In this example, flow shops 1 and 2 need to be further processed, but flow shop 3 doesn't since all its jobs have been scheduled.

Step 17: Select the third job from the sorted list in Step 11. Generate the three possible partial schedules by inserting the job in the partial schedule kept in the last run. Results are shown as follows:

$$
\begin{aligned}
& \text { Flow shop1: possible partial schedules }=\left\{\begin{array}{l}
J_{2}, J_{5}, J_{3} \\
J_{5}, J_{3}, J_{2} \\
J_{5}, J_{2}, J_{3}
\end{array}\right\} \text {, and } \\
& \text { Flow shop2: possible partial schedules }=\left\{\begin{array}{l}
J_{4}, J_{8}, J_{1} \\
J_{8}, J_{1}, J_{4} \\
J_{8}, J_{4}, J_{1}
\end{array}\right\} .
\end{aligned}
$$

Step 18: Calculate the total completion time of each possible partial schedule generated in Step 17. Results are shown in Table 7.

Table 6. The completion times of the two partial schedules in each flow shop Flow shop 1:

Case 1 


\begin{tabular}{||c|c|c|c||}
\hline$J o b_{i}$ & $f_{11}$ & $f_{21}$ & $f_{31}$ \\
\hline$J_{5}$ & 5 & 10 & 16 \\
\hline$J_{3}$ & 10 & 12 & 20 \\
\hline
\end{tabular}

Case 2

\begin{tabular}{||c|c|c|c||}
\hline \hline$J o b_{i}$ & $f_{11}$ & $f_{21}$ & $f_{31}$ \\
\hline$J_{3}$ & 5 & 7 & 11 \\
\hline$J_{5}$ & 10 & 15 & 21 \\
\hline
\end{tabular}

Flow shop 2:

Case 1

\begin{tabular}{||c|c|c|c||}
\hline \hline$J_{o b}$ & $f_{11}$ & $f_{21}$ & $f_{31}$ \\
\hline$J_{1}$ & 4 & 11 & 14 \\
\hline$J_{8}$ & 7 & 17 & 20 \\
\hline
\end{tabular}

Case 2

\begin{tabular}{||c|c|c|c||}
\hline \hline$J_{o b_{i}}$ & $f_{11}$ & $f_{21}$ & $f_{31}$ \\
\hline$J_{8}$ & 3 & 9 & 12 \\
\hline$J_{1}$ & 7 & 16 & 19 \\
\hline
\end{tabular}

Flow shop 3:

Case 1
\begin{tabular}{||c|c|c|c|}
\hline \hline$J o b_{i}$ & $f_{11}$ & $f_{21}$ & $f_{31}$ \\
\hline$J_{6}$ & 7 & 10 & 14 \\
\hline$J_{7}$ & 11 & 15 & 20 \\
\hline
\end{tabular}

Case 2

\begin{tabular}{||c|c|c|c||}
\hline \hline$J_{o b_{i}}$ & $f_{11}$ & $f_{21}$ & $f_{31}$ \\
\hline$J_{7}$ & 4 & 8 & 13 \\
\hline$J_{6}$ & 11 & 14 & 18 \\
\hline
\end{tabular}

Step 19: Keep the partial schedule with the minimum total completion time. Results are shown as Flow shop1: partial schedule $=\left\{J_{2}, J_{5}, J_{3}\right\}$, and Flow shop2: partial schedule $=\left\{J_{4}, J_{8}, J_{1}\right\}$.

Step 20: Set $k=k+1=4$. Since all the jobs in the sorted lists in flow shops 1 and 2 are processed, Step 21 is then done.

Table 7. The total completion time of each possible partial schedule for $k=3$

Flow shop 1: 


\begin{tabular}{|c|c|c|c||}
\hline$J_{o b_{i}}$ & $f_{11}$ & $f_{21}$ & $f_{31}$ \\
\hline$J_{2}$ & 1 & 6 & 8 \\
\hline$J_{5}$ & 6 & 11 & 17 \\
\hline$J_{3}$ & 11 & 13 & 21 \\
\hline
\end{tabular}

Case 2

\begin{tabular}{||c|c|c|c||}
\hline \hline$J o b_{i}$ & $f_{11}$ & $f_{21}$ & $f_{31}$ \\
\hline$J_{5}$ & 5 & 10 & 16 \\
\hline$J_{3}$ & 10 & 12 & 20 \\
\hline$J_{2}$ & 11 & 17 & 22 \\
\hline
\end{tabular}

Case 3

\begin{tabular}{||c|c|c|c||}
\hline \hline$J o b_{i}$ & $f_{11}$ & $f_{21}$ & $f_{31}$ \\
\hline$J_{5}$ & 5 & 10 & 16 \\
\hline$J_{2}$ & 6 & 15 & 18 \\
\hline$J_{3}$ & 11 & 17 & 22 \\
\hline
\end{tabular}

Flow shop 2:

Case 1

\begin{tabular}{||c|c|c|c||}
\hline \hline$J o b_{i}$ & $f_{11}$ & $f_{21}$ & $f_{31}$ \\
\hline$J_{4}$ & 2 & 7 & 10 \\
\hline$J_{8}$ & 5 & 13 & 16 \\
\hline$J_{1}$ & 9 & 20 & 23 \\
\hline
\end{tabular}

Case 2

\begin{tabular}{||c|c|c|c||}
\hline \hline$J_{o b_{i}}$ & $f_{11}$ & $f_{21}$ & $f_{31}$ \\
\hline$J_{8}$ & 3 & 9 & 12 \\
\hline$J_{1}$ & 7 & 16 & 19 \\
\hline$J_{4}$ & 9 & 21 & 24 \\
\hline
\end{tabular}

Case 3

\begin{tabular}{||c|c|c|c||}
\hline \hline$J_{o b}$ & $f_{11}$ & $f_{21}$ & $f_{31}$ \\
\hline$J_{8}$ & 3 & 9 & 12 \\
\hline$J_{4}$ & 5 & 14 & 17 \\
\hline$J_{1}$ & 9 & 21 & 24 \\
\hline
\end{tabular}

Step 21: The scheduled flow shops $Q_{F_{1}}, Q_{F_{2}}, Q_{F_{3}}$ are formed as follows:

Flow shop1: $Q_{F_{1}}=\left\{J_{2}, J_{5}, J_{3}\right\}$, 
Flow shop2: $Q_{F_{2}}=\left\{J_{4}, J_{8}, J_{1}\right\}$, and

Flow shop3: $Q_{F_{3}}=\left\{J_{7}, J_{6}\right\}$.

Set the final completion time of each flowshop $f_{i}=$ the completion time of the $m$-th machine $f_{m i}$. The results are listed in Table 8 .

Table 8. The completion time of each flowshop

\begin{tabular}{||c|c||}
\hline \hline Flow shop & Completion time $\left(f_{i}\right)$ \\
\hline$F_{1}$ & 21 \\
\hline$F_{2}$ & 23 \\
\hline$F_{3}$ & 18 \\
\hline
\end{tabular}

Step 22: Find the maximal final completion time ff among the completion time of all the flow-shops. We can thus get:

$$
f f=23 .
$$

$\mathrm{ff}$ is then output as the final total completion time, and the final schedule is shown in Figure 1.

Machine Center 1

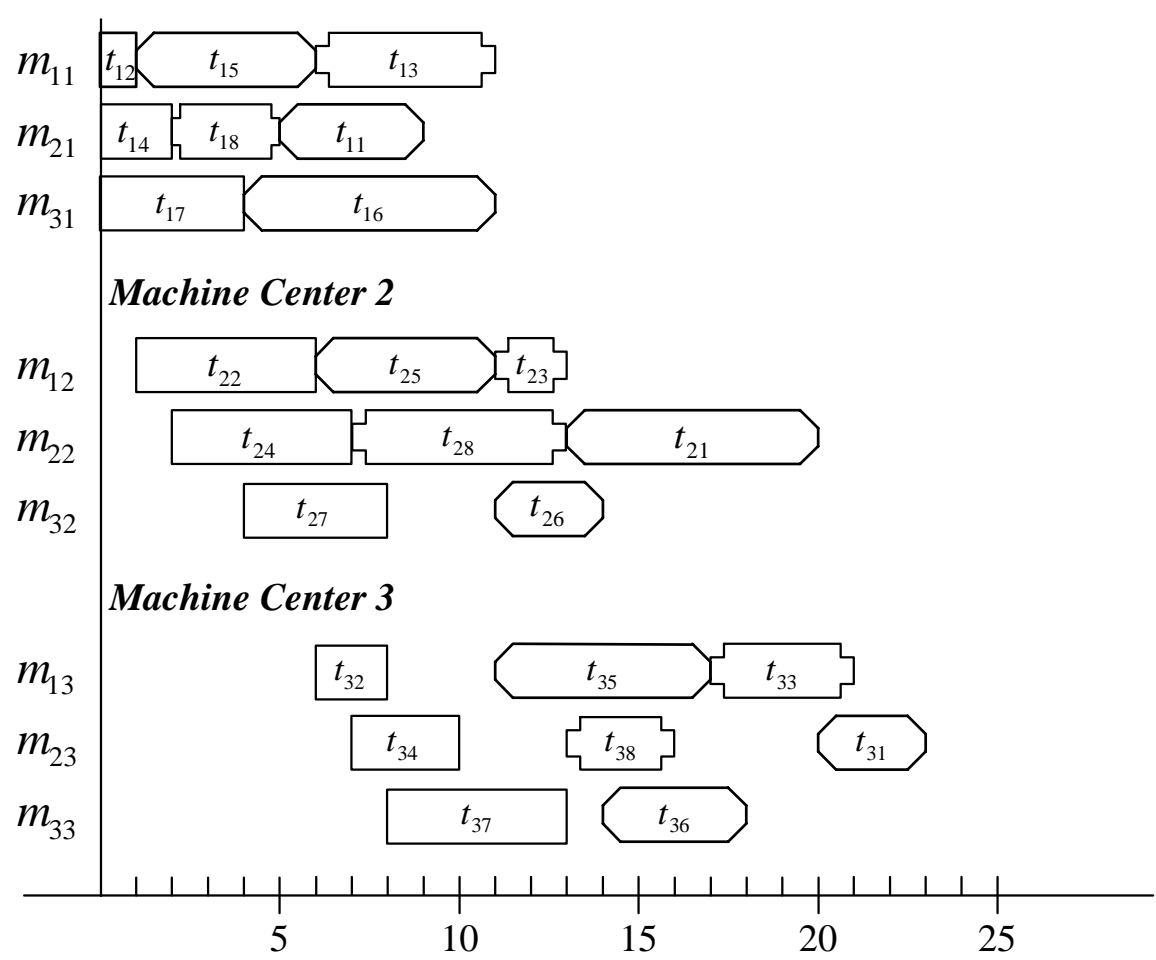

Figure 1: The final scheduling result in the example 


\section{Experiments}

This section reports on experiments made to show the performance of the proposed scheduling algorithm for flexible flowshop problems of more than two machine centers. They were implemented by Visual C++ at a AMD Athlon(tm) XP 1800+ PC. Also presented are experiments made to compare the time required by the proposed algorithm with that required by the A-star approach shown in [8]. Five sets of problems were tested, respectively for 3 to 7 jobs. Each job has three tasks and each machine center has two homogeneous machines. The execution time of each task was randomly generated in the range of 5 to 50 . Each set of problems was executed for 20 tests and the makespans and computation times were measured.

The A-star approach considered all possible combinations and used pruning techniques to increase its efficiency. The makespans obtained in this way were thus optimal. The makespans for problems of three to seven jobs by our proposed method and by the A-star approach are shown respectively in Figures 2 to 6 . The A-star approach in [8] did not work for more than seven jobs in our environments due to the large amount of computation time.

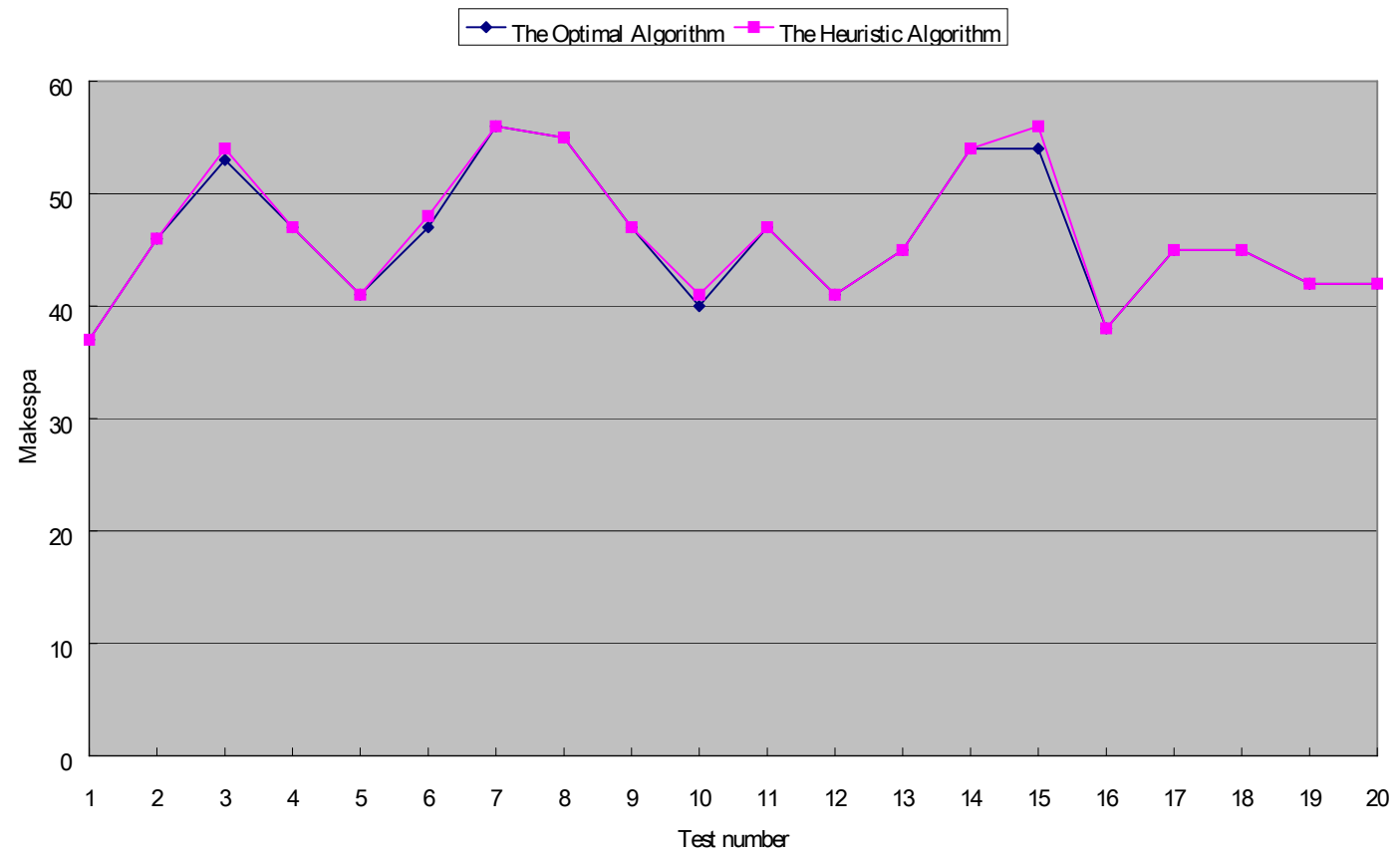

Figure 2: Makespans of 20 tests for three jobs 


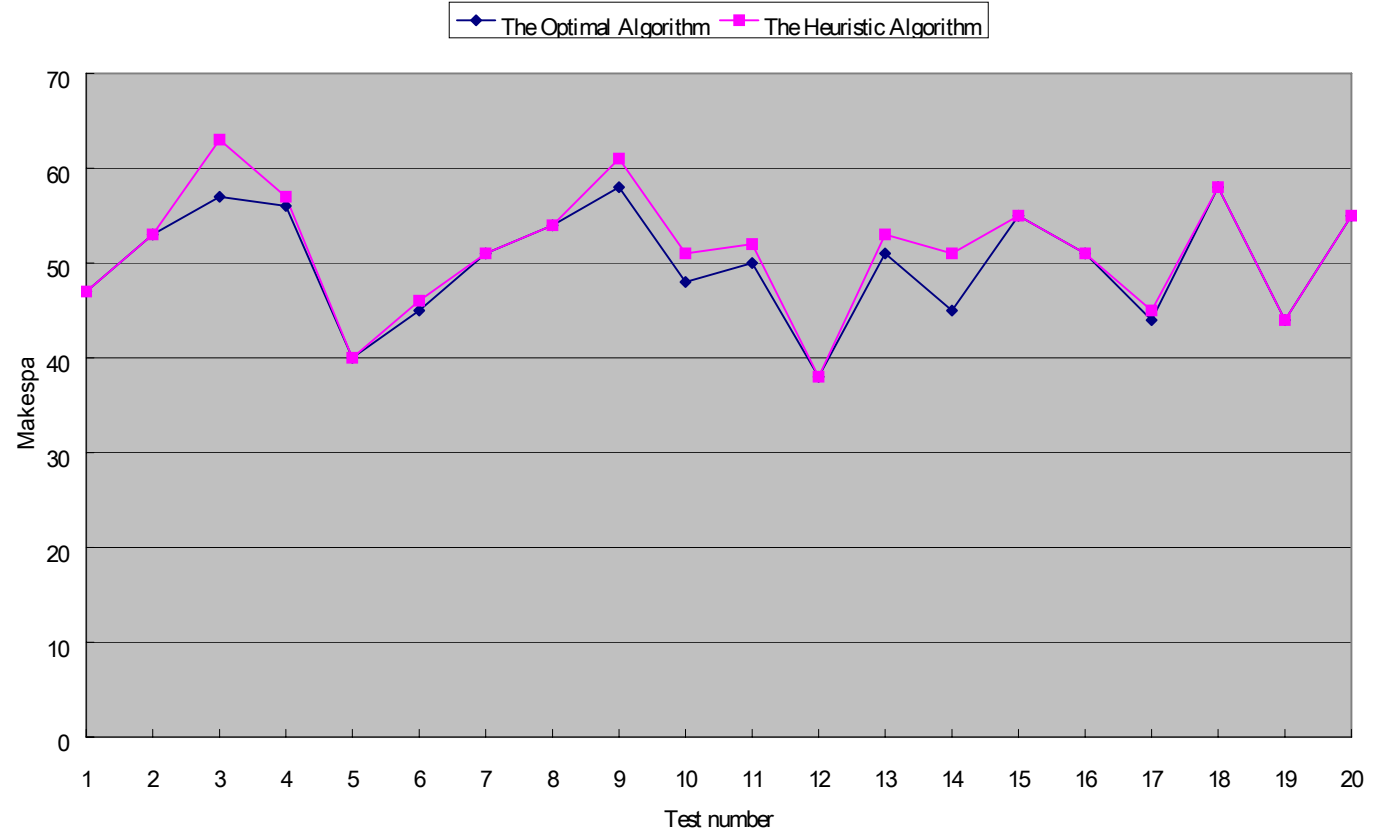

Figure 3: Makespans of 20 tests for four jobs

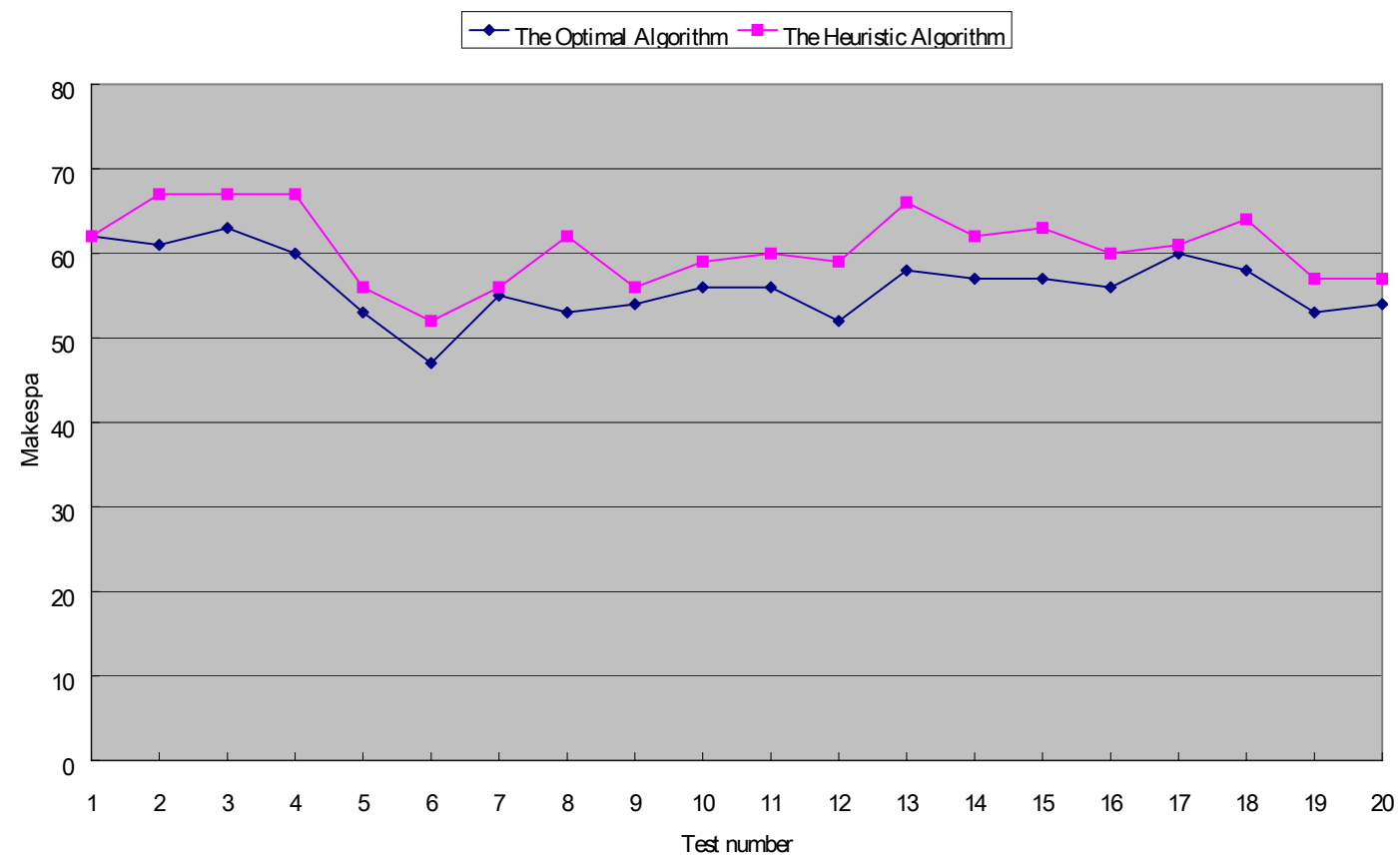

Figure 4: Makespans of 20 tests for five jobs 
The Optimal Algorithm - The Heuristic Algorithm

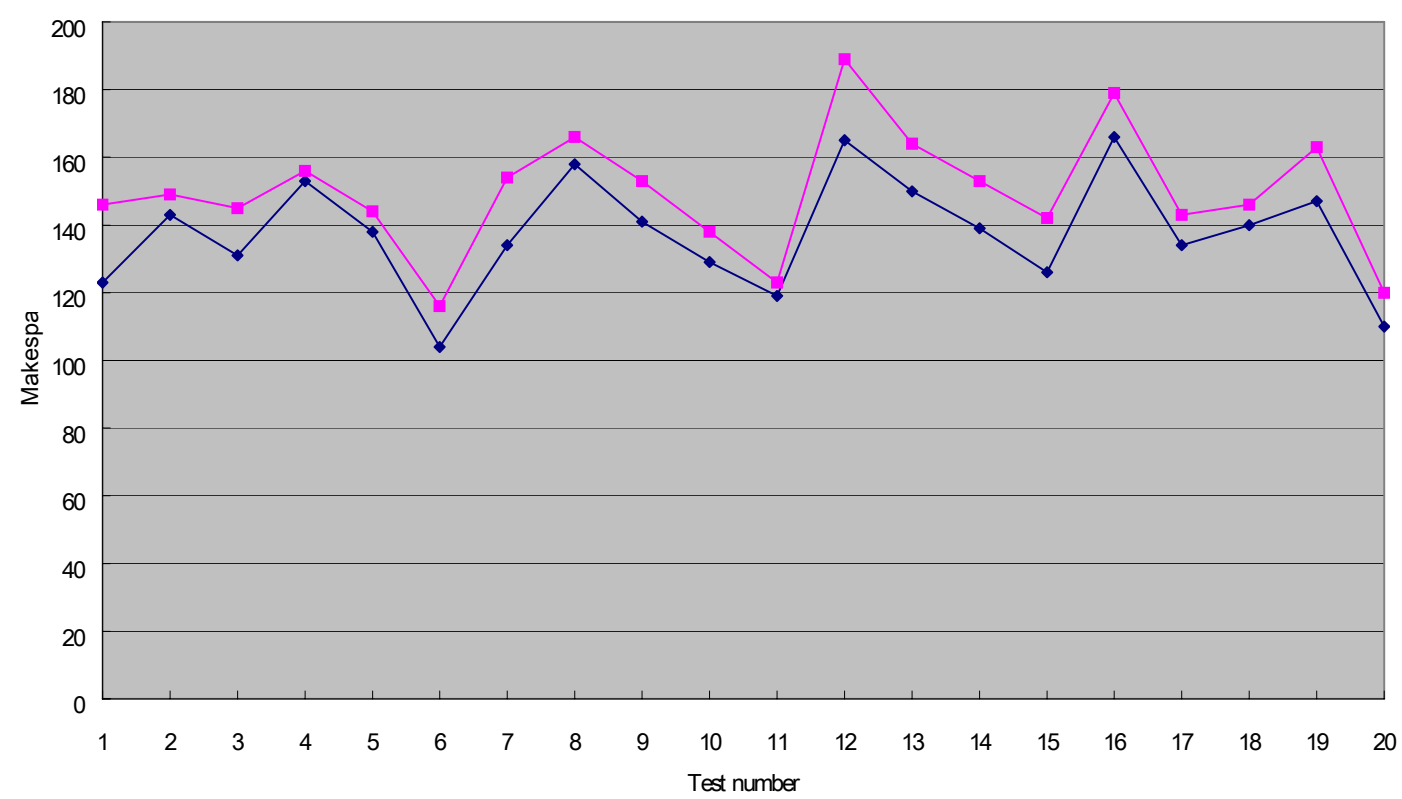

Figure 5: Makespans of 20 tests for six jobs

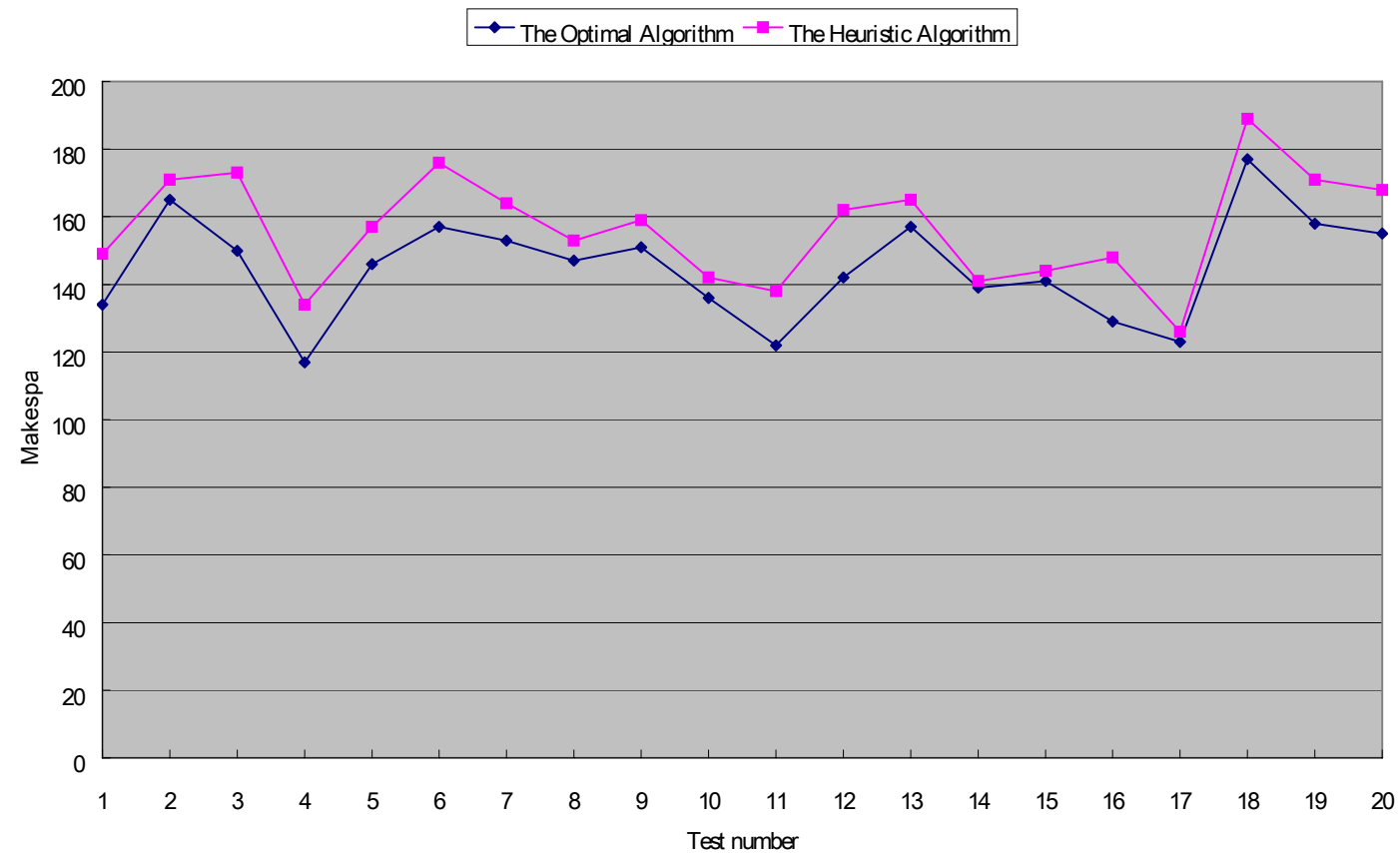

Figure 6: Makespans of 20 tests for seven jobs 
Solving flexible flow-shop problems

The average error rates of different numbers of jobs by our proposed algorithm are shown in Table 9.

Table 9. The average error rates of different numbers of jobs by the proposed algorithm

\begin{tabular}{||c|c||}
\hline Job number & Average error rate (\%) \\
\hline 3 & 0.5109078 \\
\hline 4 & 2.4742148 \\
\hline 5 & 7.8981798 \\
\hline 6 & 8.786217 \\
\hline 7 & 8.0867188 \\
\hline
\end{tabular}

The average CPU times for problems of three to seven jobs are shown in Figure 7. The A-star approach cannot run over seven jobs due to the high time complexity. The average CPU times for problems of eight to twenty-five jobs by our proposed algorithm are also shown there.

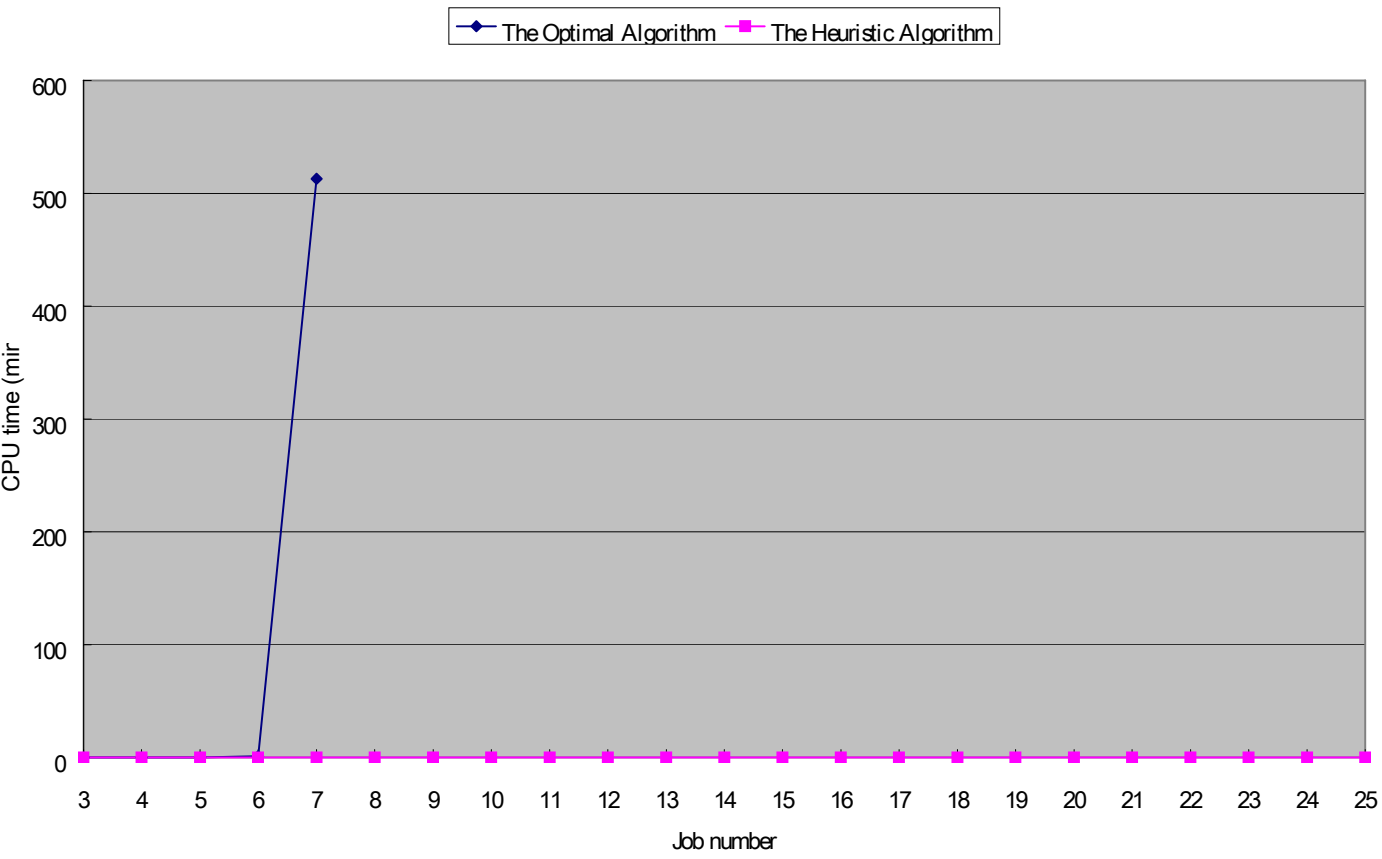

Figure 7: The average CPU times for processing different numbers of jobs

From the above figures, it is easily seen that our proposed algorithm got a little larger makespans than the A-star approach did. The computational time needed by the A-star approach was, however, much larger than that needed by our proposed algorithm, especially when the job number was large. Actually, since the flexible flow-shop problem is an NP-hard problem, the A-star approach can work only for a small number of jobs. Our proposed algorithm can solve this problem, 
thus being more suitable for real applications.

\section{Conclusion}

Appropriate scheduling cannot only reduce manufacturing costs but also reduce the possibility of violating due dates. Finding good schedules for given sets of jobs can thus help factory supervisors control job flows and provide for nearly optimal job sequencing.

Scheduling jobs in flexible flow shops has long been known an NP-complete problem. In this paper, we have proposed an LPT_LN scheduling algorithm for scheduling jobs in flexible flow shops with more than two machine centers. The proposed algorithm needs much less computational time, but only with a little larger makespans than the A-star approach did. In the future, we will consider other task constraints, such as setup times, due dates, and priorities.

\section{References}

[1] S. C. Chung and D. Y. Liao, Scheduling flexible flow shops with no setup effects, The 1992 IEEE International. Conference on Robotics and Automation, 1992, 1179 $-1184$.

[2] R. A. Dudek, S. S. Panwalkar and M. L. Smith, The lessons of flowshop scheduling research, Operations Research, 40 (1992) 7 - 13.

[3] T. P. Hong, C. M Huang and K. M. Yu, LPT scheduling for fuzzy tasks, Fuzzy Sets and Systems, 97 (1998), 277 - 286.

[4] R. Logendran and N. Nudtasomboon, Minimizing the makespan of a group scheduling problem: a new heuristic, International Journal of Production Economics, 22 (1991) $217-230$.

[5] T. E. Morton and D. W. Pentico, Heuristic Scheduling Systems with Applications to Production Systems and Project Management, Wiley, New York, 1993.

[6] J. Schaller, A new lower bound for the flow shop group scheduling problem, Computers and Industrial Engineering, 41 (2001), 151 - 161.

[7] C. Sriskandarajah and S. P. Sethi, Scheduling algorithms for flexible flow shops: worst and average case performance, European Journal of Operational Research, 43 (1989), 143 - 160.

[8] C. L. Wang, Several Heuristic Scheduling Algorithms for Solving Flexible Flow-Shop Problems, Master Thesis, Department of Information Engineering, I-Shou University, Taiwan, 2001.

[9] D. L. Yang, M. S. Chern, Two-machine flowshop group scheduling problem, Computers \& Operations Research, 27 (2000), 975 - 985.

Received: July 30, 2005 